\title{
Fractured aluminum nasopharyngeal swab during drive-through testing for COVID-19: radiographic detection of a retained foreign body
}

\author{
Antoine Azar ${ }^{1} \cdot$ Daniel E. Wessell $^{1} \cdot$ Jeffrey R. Janus ${ }^{2} \cdot$ Leslie V. Simon $^{3}$ \\ Received: 23 July 2020 / Revised: 6 August 2020 / Accepted: 9 August 2020 / Published online: 21 August 2020 \\ (C) ISS 2020
}

\begin{abstract}
The ongoing coronavirus disease 2019 (COVID-19) pandemic has increased the need for safe and efficient testing as a key containment strategy. Drive-through testing with nasopharyngeal swab has been implemented in many places in the USA as it allows for expeditious testing of large numbers of patients, limits healthcare workers' risk of exposure, and minimizes the use of personal protective equipment. We present a case where the aluminum shaft of the nasopharyngeal swab fractured during specimen collection at a drive-through testing facility and was suspected to have remained in the asymptomatic patient. Initial evaluation with a series of radiographs covering the skull base, neck, chest, and abdomen did not reveal the swab. On further clinical evaluation, the swab was found endoscopically, lodged between the left inferior turbinate and nasal floor, and was removed by an otorhinolaryngologist. Using a phantom model, we aimed to delineate an imaging technique to better visualize the aluminum shaft of the nasopharyngeal swab on radiographs to help in identification. A technique using lower tube voltage $(\mathrm{kVp})$ with tight collimation centered at the nasal bones area produced the best visualization of the aluminum shaft of the swab. Recognition that aluminum foreign bodies may be difficult to visualize radiographically and optimization of radiograph acquisition technique may help guide clinical management in unusual cases. Further evaluation with computed tomography or endoscopy should be considered in suspected cases where radiographs are negative.
\end{abstract}

Keywords COVID-19 · Nasopharyngeal swab $\cdot$ Radiographs $\cdot$ X-ray $\cdot$ Aluminum $\cdot$ Foreign body

\section{Introduction}

The USA has performed millions of tests for SAR-CoV-2, the virus that causes COVID-19. Drive-through testing was

Antoine Azar

Azar.Antoine@mayo.edu

Daniel E. Wessell

Wessell.Daniel@mayo.edu

Jeffrey R. Janus

Janus.Jeffrey@mayo.edu

Leslie V. Simon

Simon.Leslie@mayo.edu

1 Department of Radiology, Mayo Clinic, 4500 San Pablo Road S, Jacksonville, FL 32224, USA

2 Department of Otorhinolaryngology, Mayo Clinic, 4500 San Pablo Road S, Jacksonville, FL 32224, USA

3 Department of Emergency Medicine, Mayo Clinic, 4500 San Pablo Road S, Jacksonville, FL 32224, USA first implemented in South Korea [1] but is now used throughout the world to efficiently test large numbers of patients as the cornerstone of successful containment strategies [2]. The entire process takes less than 10 minutes for each patient and can be performed without the patient ever having to leave their car. This allows for minimal usage of personal protective equipment and minimalizes contact time between potentially infectious patients and healthcare workers [3]. Our healthcare organization has performed over 100,000 nasopharyngeal swab tests for COVID-19 at our drive-through testing centers in 4 different states. We present a complication that occurred during nasopharyngeal swab collection where the aluminum shaft of the swab fractured and was suspected to have remained in the asymptomatic patient. The swab went undetected radiographically but was later found to be lodged in the patient's nasal cavity on endoscopic examination and was removed by an otorhinolaryngologist. We discuss imaging strategies to help in radiographic identification of aluminum foreign bodies such as many of the nasopharyngeal swabs that are widely used during the COVID-19 pandemic. 
Fig. 1 Frontal view (a) and lateral view (b) radiographs obtained in the emergency department covering the skull base and neck. Note the radiopaque bridge (vertical arrow) and the staples (horizontal arrow) of the face mask the patient was wearing
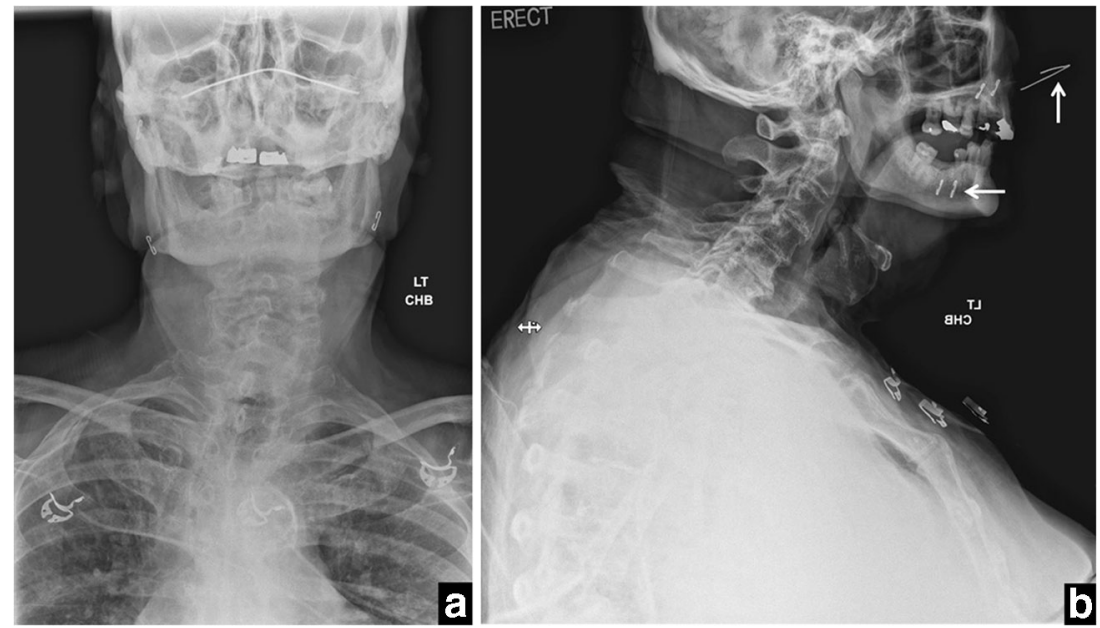

\section{Case report}

This patient is a 99-year-old Caucasian male with an extensive past medical history which included hypertension, diabetes, coronary artery disease, osteopenia, and prior stroke who was driven to the drive-through testing facility by a family member to be tested for COVID-19 infection. The patient presented for testing with a history of increased cough for several days that was worse at night. The cough was intermittently productive with discolored mucous. He specifically denied fever, chills, diarrhea, chest pain, shortness of breath, or loss of taste or smell. As per the usual testing protocol, a BBL ${ }^{\mathrm{TM}}$ rayon-tipped aluminum shaft CultureSwab TM was inserted into the left nostril by a nurse who had performed the procedure several hundred times. When the nurse attempted to withdraw the swab, it broke off. Careful inspection of the patient's nose, posterior pharynx, clothing, and the inside of the car did not reveal the missing portion of the swab at the testing site. The nurse was certain that the swab was intact before it was inserted into the patient. The patient denied foreign body sensation, difficulty breathing, or difficulty swallowing and was referred to the emergency department for further evaluation.

In the emergency department, the patient remained asymptomatic and denied foreign body sensation. He was afebrile with an oral temperature of $38.3{ }^{\circ} \mathrm{C}$, with oxygen saturation of $95 \%$ on room air and respiratory rate of 16 breaths per minute. Repeat inspection of the nose and posterior pharynx using an otoscope and nasal speculum did not reveal the fractured swab. The lungs were clear, heart rate and rhythm were normal, and the remainder of the exam was unremarkable. Radiographs covering the skull base and neck soft tissues (Fig. 1a and b) and radiographs of the abdomen did not show any evidence of radiopaque foreign body by the emergency physician's read which was confirmed by the over reads performed by a radiologist. Chest X-ray did not show foreign body or pneumonia. Because the location of the swab was still unclear, the patient was then referred to the otorhinolaryngology clinic. Nasal speculum exam again revealed normal mucosa without evidence of deviation of the nasal septum. The turbinates appeared normal as did the oral cavity, soft and hard palate, tonsils, and posterior oropharynx. There was no evidence of erythema, mucosal injury, or foreign body. The patient was then consented for nasal endoscopy to ensure that there was no foreign body in the nasal cavity or paranasal sinuses that was not visible by anterior rhinoscopy. Cotton pledgets soaked in $0.05 \%$ oxymetazoline and $4 \%$ topical lidocaine were placed in both nasal cavities. Pledgets were placed in lieu of the traditional lidocaine/oxymetazoline spray to reduce the generation of aerosol in the setting of possible COVID-19 infection. After topical anesthesia, a 0 degree endoscope was advanced into the left nasal cavity revealing a foreign body firmly wedged under the inferior turbinate. The object was oriented with the distal aspect of the swab in the nasopharynx, the mid-portion in the inferior meatus, and the fractured portion pinned between the inferior turbinate and the nasal floor. An alligator forceps was then used to remove the fractured swab which measured nearly $5 \mathrm{~cm}$ (Fig. 2). There were no complications.

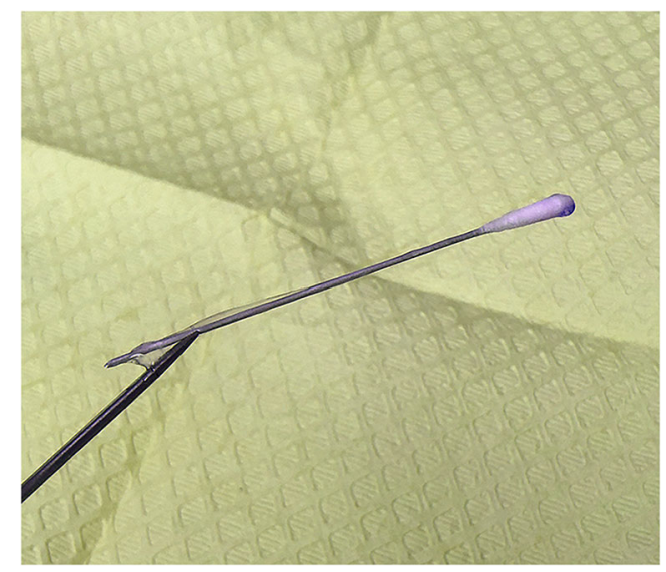

Fig. 2 Photograph of the nasopharyngeal swab after endoscopic removal 


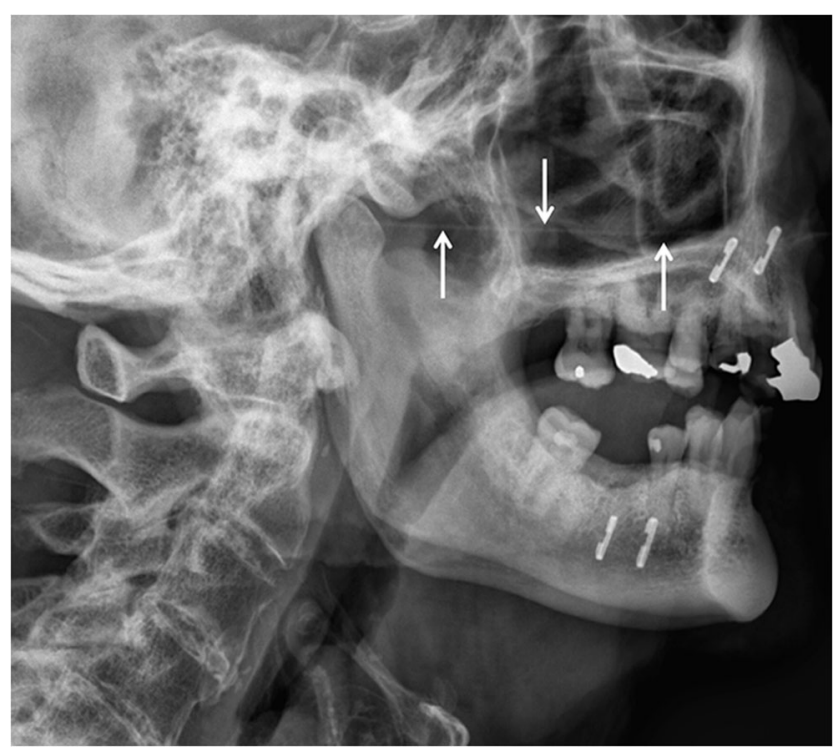

Fig. 3 Magnification of the lateral view radiograph obtained in the emergency department covering the skull base and neck showing a thin minimally radiopaque line in the nasal cavity representing the retained nasopharyngeal swab (arrows)

\section{Discussion}

In the era of COVID-19, large numbers of patients are tested with nasopharyngeal swabs at drive-through locations remote to the hospital setting. Swab fracture with retained foreign body is unusual but has been reported in recent months as a possible complication $[4,5]$. The types of nasopharyngeal swabs used to collect the specimens for COVID-19 testing may vary; however, FDA-authorized instructions for several of the widely used tests recommend specimen collection using swabs with aluminum or plastic shafts [6]. In this case, the shaft of the swab was made of aluminum.

While the nasopharyngeal swab went undetected on initial radiographic evaluation, review of the lateral radiographs that covered the skull base and neck soft tissues in retrospect did

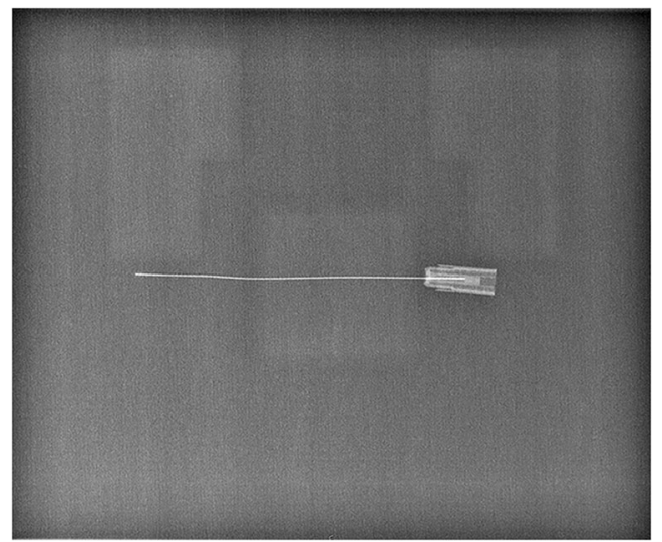

Fig. 4 Radiograph of a standard standard BBL TM rayon-tipped aluminum shaft CultureSwab TM obtained at $66 \mathrm{kVp}$ and $1 \mathrm{mAs}$ reveal a minimally radiopaque thin line at the inferior aspect of the nasal cavity representing the aluminum shaft of the swab (Fig. 3). For this radiograph, exposure was set at $65.9 \mathrm{kVp}$ and $28 \mathrm{mAs}$, and the field of view extended from the skull base to the upper chest without collimation. Notably, the patient was wearing a surgical-type face mask during the examination, and the bridge piece and staples retaining the bands of the mask were easily visualized as dense radiopaque objects.

The visibility of aluminum foreign bodies on radiographs has long been an area of interest to radiologists. The adoption of aluminum can pull tabs by soft drink manufacturers in the late
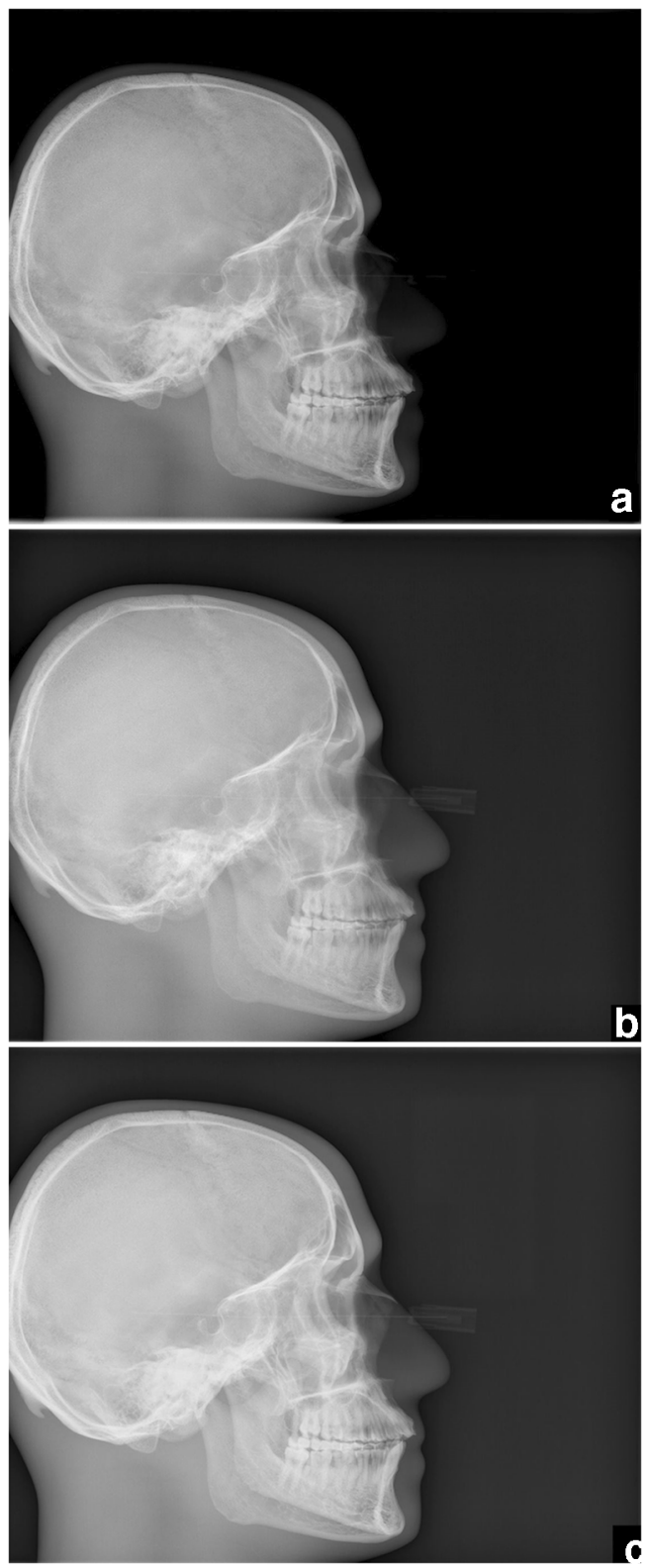

Fig. 5 Uncollimated radiographs of a phantom model with aluminum nasopharyngeal swab obtained with tube voltage of $66 \mathrm{kVp}(\mathbf{a})$, $121 \mathrm{kVp}(\mathbf{b})$, and $56 \mathrm{kVp}(\mathbf{c})$ 
twentieth century introduced the issue of identifying accidentally swallowed can tabs on radiographs, which often went undetected as has been reported several times in children $[7,8]$ and in adults $[9,10]$. In one such case, the can tab was not visible on radiographs but was later identified on computed tomography (CT) [9]. Additional cases have been reported of aluminum-based foreign bodies that are not readily detected radiographically, including aluminum-jacketed ammunition and Japanese 1-yen coins [11, 12]. Interestingly, a 1973 proposal by the US Mint to replace the copper penny with a cheaper aluminum alloy alternative was eventually denied due to concerns raised by physicians that ingested coins, a commonly encountered issue in children, may also not be detectable with X-ray [13, 14]. In a study by Ellis in 1993 where aluminum pieces of different sizes were implanted in chicken wings to determine if they are radiopaque, the study author concluded that the pieces were radiographically visible in soft tissue but warned that superimposition of bony structures may make detection more difficult [15]. Similarly, in a 2005 study by Valente et al., aluminum can tabs were placed in the posterior pharynx and upper esophagus of cadavers, and radiographs were obtained to be assessed by two radiologists blinded to the knowledge of which cadavers contained the tabs and the number of tabs present. The study authors concluded that while the aluminum foreign bodies were often detected, the sensitivity of detection was not adequate to rule out their presence if not seen [16]. Although not recommended as a primary modality for detection of foreign bodies, $\mathrm{CT}$ has been demonstrated superior to radiographs at detection of inhaled or ingested radiolucent foreign bodies and has been recommended in situations when a suspected aluminum foreign body is not detected radiographically $[9,17]$.

The difficulty with radiographic imaging of aluminum stems from its physical properties whereby its ability to attenuate diagnostic $\mathrm{X}$-rays through photoelectric absorption is intermediate compared with soft tissue and bone. This is likely further exacerbated by the use of aluminum filtration in some modern X-ray machines to improve image quality and minimize radiation dose, which effectively eliminates the photons just above the k-edge of aluminum that would give the best contrast between aluminum and soft tissue from the X-ray beam [10].

Recognizing this, we can tailor the radiograph technique in our advantage to give the best chance for detection of a suspected aluminum foreign body such as a nasopharyngeal swab. We therefore performed an experiment to identify the best imaging technique to visualize an aluminum shaft nasopharyngeal swab used for COVID-19 drive-through testing. First, we image a standard BBL TM rayon-tipped aluminum shaft CultureSwab TM at $66 \mathrm{kVp}$ and $1 \mathrm{mAs}$ (Fig. 4). Then, using a phantom model, we taped the swab onto the side of a skull phantom at the level of the nasal cavity (note that the phantom has no nasal cavity to place the swab into) and obtained radiographs covering the entire skull without collimation with exposure of $66 \mathrm{kVp}$ and $5 \mathrm{mAs}$ (Fig. 5a), $121 \mathrm{kVp}$ and $1 \mathrm{mAs}$ (Fig. 5b), and $56 \mathrm{kVp}$ and $10 \mathrm{mAs}$ (Fig. 5c). We then collimated the field of view to the nasal bones and obtained additional radiographs at $121 \mathrm{kVp}$ and $3 \mathrm{mAs}$ (Fig. 6a) and at $56 \mathrm{kVp}$ and $77 \mathrm{mAs}$ (Fig. 6b). Those parameters were chosen to illustrate the effect of high versus low tube voltage $(\mathrm{kVp})$ and collimation on swab conspicuity. The parameters matched the tube voltage used for the clinical radiograph that was obtained in the emergency department $(66 \mathrm{kVp})$ and the tube voltage routinely used for chest radiographs $(121 \mathrm{kVp})$ and for extremity radiographs $(56 \mathrm{kVp})$ at our institution. The mAs was adjusted to provide similar exposure and noise in the radiographs. After review, it was clear that the collimated images obtained at lower tube voltages produced the best visualization of the aluminum shaft of the nasopharyngeal swab. Collimation reduces scatter, which in turn improves image contrast and shaft conspicuity. Low tube voltage results in lower mean energy of the $\mathrm{X}$-ray beam, which increases the probability of photoelectric interaction with the swab shaft and thus also improves contrast and swab conspicuity.

Fig. 6 Collimated radiographs of the phantom model with aluminum nasopharyngeal swab obtained with tube voltage of $121 \mathrm{kVp}(\mathbf{a})$ and $56 \mathrm{kVp}(\mathbf{b})$
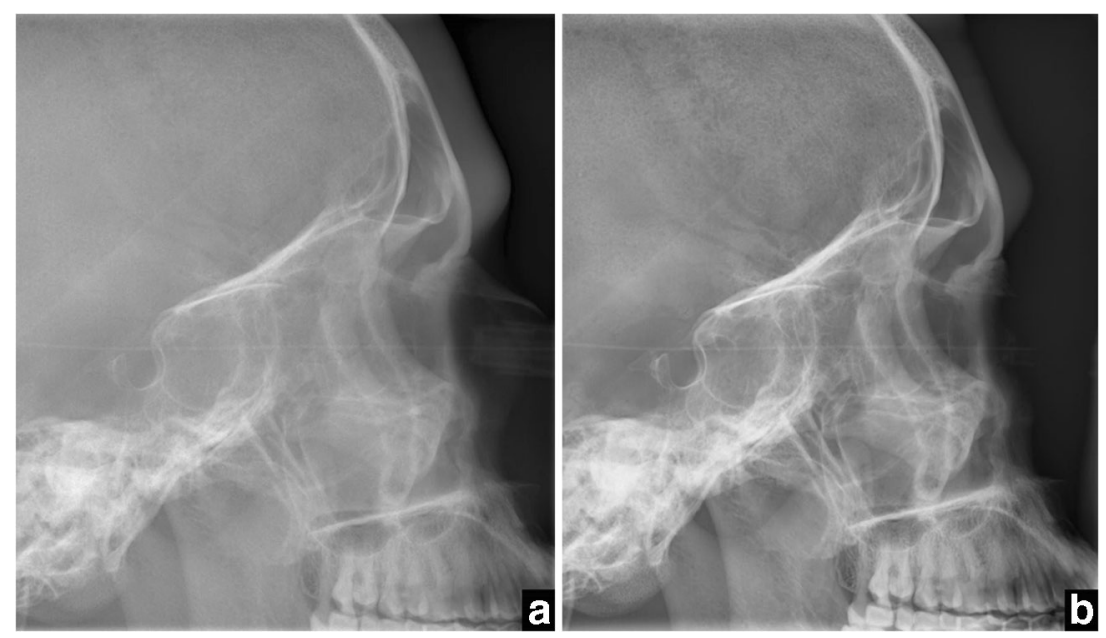
Recognition that aluminum foreign bodies may be difficult to visualize radiographically and optimization of the radiograph acquisition technique could play a key role in management of unusual cases of nasopharyngeal swab fracture with suspected ingested, aspirated, or retained nasopharyngeal foreign body, which may be asymptomatic. In the setting of negative radiographs, further evaluation should be considered with CT imaging or endoscopy, as was critical in this case to prevent complications.

Acknowledgments Thank you to LuAnne Williams, MSN, RN for her insight on the drive-through testing process, Cheryl Jackson, PA-C for her initial evaluation in the otorhinolaryngology clinic, and Kathy Pastor, RT and Craig Hildestad, RT for their invaluable help in performing the phantom model experiments.

Authors' contributions (I) Conception and design: L.S. and D.W. (II) Collection, analysis, and presentation of case report information and images: All authors. (III) Drafting of the article: All authors. (IV) Critical revision of the article: All authors. (V) Final approval of manuscript: All authors.

\section{Compliance with ethical standards}

Conflict of interest The authors declare that they have no conflict of interest.

\section{References}

1. Kwon KT, Ko JH, Shin H, Sung M, Kim JY. Drive-through screening center for COVID-19: a safe and efficient screening system against massive community outbreak. J Korean Med Sci. 2020;35(11):e123.

2. Cheng MP, Papenburg J, Desjardins M, Kanjilal S, Quach C, Libman M, et al. Diagnostic testing for severe acute respiratory syndrome-related coronavirus 2: a narrative review. Ann Intern Med. 2020;172(11):726-34.
3. Ton AN, Jethwa T, Waters K, Speicher LL, Francis D. COVID-19 drive through testing: an effective strategy for conserving personal protective equipment. Am J Infect Control. 2020;48(6):731-2.

4. Medas R, Coelho R, Macedo G. An unusual collateral damage of COVID-19 pandemic. Gastrointest Endosc. 2020.

5. Mughal Z, Luff E, Okonkwo O, Hall CEJ. Test, test, test - a complication of testing for coronavirus disease 2019 with nasal swabs. J Laryngol Otol 2020:1-4.

6. Centers for Disease Control and Prevention, Division of Viral Diseases. CDC 2019-nCoV real-time RT-PCR diagnostic panel instructions for use. CDC publication CDC-006-00019. https:// www.fda.gov/media/134922/download. Accessed August 5, 2020.

7. Eggli KD, Potter BM, Garcia V, Altman RP, Breckbill DL. Delayed diagnosis of esophageal perforation by aluminum foreign bodies. Pediatr Radiol. 1986;16(6):511-3.

8. Donnelly LF. Beverage can stay-tabs: still a source for inadvertently ingested foreign bodies in children. Pediatr Radiol. 2010;40(9): 1485-9.

9. Kotsenas AL, Campeau NG, Oeckler RA, Kuzo RS. Evaluation of suspected aspirated beverage can pull tab: radiographs may not be enough. Case Rep Radiol. 2014;2014:196960.

10. Stewart GD, Lakshmi MV, Jackson A. Aluminium ring pulls: an invisible foreign body. J Accid Emerg Med. 1994;11(3):201-3.

11. Conradi SE. New aluminum-jacketed ammunition: the case of the "invisible" jacket. Am J Forensic Med Pathol. 1982;3(2):153-5.

12. Takahashi J, Shiga T, Funakoshi H. Oesophageal coins invisible on chest radiography: a case report. Int J Emerg Med. 2017;10(1):27.

13. Heller RM, Reichelderfer TE, Dorst JP, Oh KS. The problem with the replacement of copper pennies by aluminum pennies. Pediatrics. 1974;54(6):684-8.

14. Dorst JP, Reichelderfer TE, Sanders RC. Radiodensity of the proposed new penny. Pediatrics. 1982;69(2):224-5.

15. Ellis GL. Are aluminum foreign bodies detectable radiographically? Am J Emerg Med. 1993;11(1):12-3.

16. Valente JH, Lemke T, Ridlen M, Ritter D, Clyne B, Reinert SE. Aluminum foreign bodies: do they show up on $\mathrm{x}$-ray? Emerg Radiol. 2005;12(1-2):30-3.

17. Tseng HJ, Hanna TN, Shuaib W, Aized M, Khosa F, Linnau KF. Imaging foreign bodies: ingested, aspirated, and inserted. Ann Emerg Med. 2015;66(6):570-82 e575.

Publisher's note Springer Nature remains neutral with regard to jurisdictional claims in published maps and institutional affiliations. 